\title{
Research on the compensation method of Indirect Tire Pressure Monitoring under Sinusoidal Driving Condition
}

\author{
Wang Liqiang, Qi Lin, Zhang Zhe, HAN Zongqi \\ College of Vehicles and Energy, Yanshan University, Hebei, China
}

\begin{abstract}
Indirect tire pressure monitoring method (ITPMS) is based on the wheel speed signal differences indirectly infer that the change of the tire pressure. In the steering mode, there is a large yaw rate, and there is a big deviation between the four wheels' speed. The traditional indirect tire pressure monitoring method based on wheel speed comparison method is invalid. That the automobiles which is matched electronic stability control system estimate the four-wheel speed according to the yaw rate and steering wheel angle become an important method to improve the accuracy of Indirect tire pressure monitoring. Four-wheel vehicle kinematics model for turning condition is established to compensate for the wheel speed in the steering mode, avoid the misjudgment of indirect tire pressure monitoring system. The seven degree of freedom vehicle model and the four wheel speed model are established firstly, and analyse the four wheel speed change of the vehicle under the condition of a certain corner input in the simulation. Then conduct a test on the vehicle road, calculated using the four wheel speed distribution module model of vehicle wheel speed on the theoretical value of the actual value of the wheel speed compensation, so it can monitor the tire pressure in turning vehicle. When the error of the wheel speed is beyond the threshold, the wheel can be judged to be in the state of lack of gas.
\end{abstract}

\section{Introduction}

Tire pressure monitoring system is one of the most important vehicle safety systems, which can reduce vehicle safety problems and reduce traffic accidents. At present, there are two solutions to solve the vehicle tire pressure monitoring: direct tire pressure monitoring program and indirect tire pressure monitoring program. Direct tire pressure monitoring scheme installs the tire pressure sensor in the tire directly and it measures the tire pressure directly. It has the advantages of high precision and small error, and the disadvantages are also obvious. The reliability of the wireless signal transmission is poor and it has short life and it is expensive. Indirect tire pressure monitoring scheme is to estimate the value of the four tire pressure using the sensor of automobile. Indirect tire pressure monitoring system does not require additional sensor. Low cost is suitable for large-scale application, and the disadvantage is the monitoring value has large error.

At present, there are three methods for indirect tire pressure monitoring: The first method is the rotation radius method. By calculating the effective tire rotation radius to monitor tire pressure, this method uses wheel speed signal of vehicle wheel speed sensor directly. Using the Calman filter method, observe the change of wheel rotation radius, and obtain tire pressure alarm threshold through the preliminary calibration. The second method is spectrum method. By analyzing the wheel speed resonance frequency, the purpose of tire pressure monitoring is achieved. The main method of monitoring tire pressure indirectly is to estimate tire torsional stiffness indirectly by using wheel speed. At present, the most mature indirect tire pressure monitoring solution is the rotation radius method.

Indirect tire pressure monitoring system (ITPMS) is a tire pressure monitoring device based on the wheel speed sensor (ABS) wheel speed sensor [1-2]. The advantages of the indirect tire pressure monitoring system are its simple structure, low cost and easy installation. At present, the indirect tire pressure monitoring system which is installed in a car assumes that the distance of the four rolling wheels is the same as they travel. Therefore, the indirect tire pressure monitoring system can only monitor the tire pressure when the vehicle runs in a straight line. If the vehicle is turning, the vehicle yaw speed will change greatly, which will result in the wheel speed difference. At the same time, the lateral force causes the tire side deviation and affects the speed signal error. The tire pressure monitoring method based on this principle is invalid. With the application of vehicle electronic stability program system (ESP) in automobile, the inertial unit which can measure the lateral acceleration, longitudinal acceleration and yaw angular velocity of the vehicle has been popularized. The yaw speed and steering angle of car body are important for wheel speed correction of the four wheel of vehicle [3-4]. Therefore, in this paper, the rotation radius method is applied to study the failure of the tire pressure monitoring method under the steering condition. A method of tire pressure monitoring and 
compensation using steering signal and yaw angular speed signal is proposed. It can improve the traditional indirect tire pressure monitoring function, and enrich the monitoring range of the tire pressure monitoring method with rotary radius method.

Firstly, a theoretical model of four wheel speed under cornering condition is established. The main method is to obtain the steering angle, yaw angle and other signals of the vehicle through the seven degree of freedom vehicle model. Then, the four wheel speed model is established to calculate the change of the four wheel speed trend under the ideal turning condition, and the simulation results are obtained. Then, the steering wheel angle and yaw rate signal in the actual road test are input to the four wheel speed model, and the actual wheel speed is corrected. Finally, the tire pressure monitoring method of traditional pulse comparison method is used to correct the rear wheel speed and realize the tire pressure monitoring under cornering conditions.

\section{Establish a theoretical simulation model}

\subsection{The establishment of seven degree equation}

The seven degree of freedom model is used to describe the dynamic state of the vehicle in the control process and on-line state observation. The model takes into account both the difference in dynamics of the four wheels and the effects of load transfer [1]. This paper is based on the kinematic model of vehicle with seven degrees of freedom. A four wheel vehicle motion model is set up, which includes the input of vehicle steering wheel and the yaw rate of vehicle. The four wheeled vehicle motion model modeling method is shown in Figure 1.

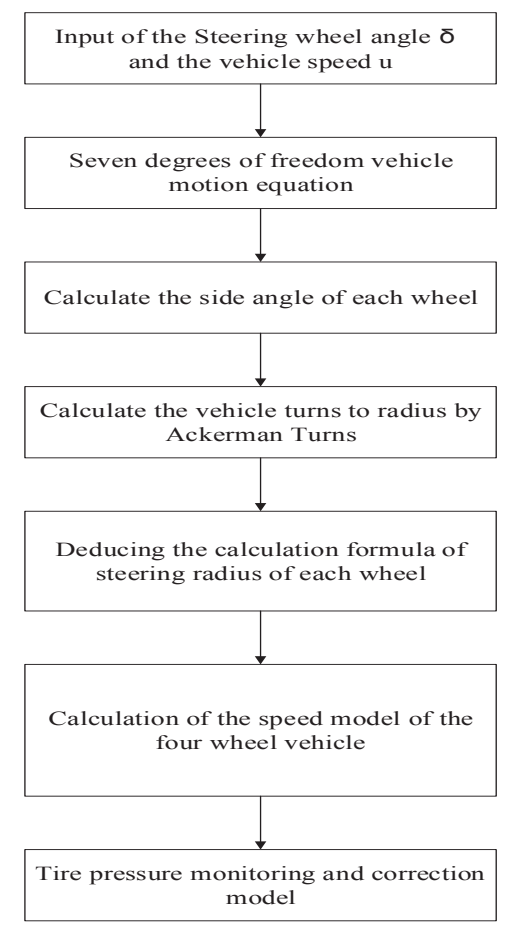

Figure 1. Modeling method for Four-wheel vehicle motion model
It is considered that the slip rate and side angle of each wheel may be inconsistent in the four wheel independent control. In the steering process and lateral load transfer. The wheel load is transferred during the braking or acceleration process, so the vertical load of the wheel is a time-varying parameter during the steering process. In addition, in the actual movement of the car, the adhesion between the tires and the pavement may be inconsistent. These factors are difficult to be quantitatively evaluated in two wheel models. Therefore, the four wheel model is needed to further describe the dynamic state of vehicle when running on a smooth road. And because of the brake intervention, the longitudinal speed of the car is time-varying, so the degree of freedom of the longitudinal motion of the car needs to be considered.

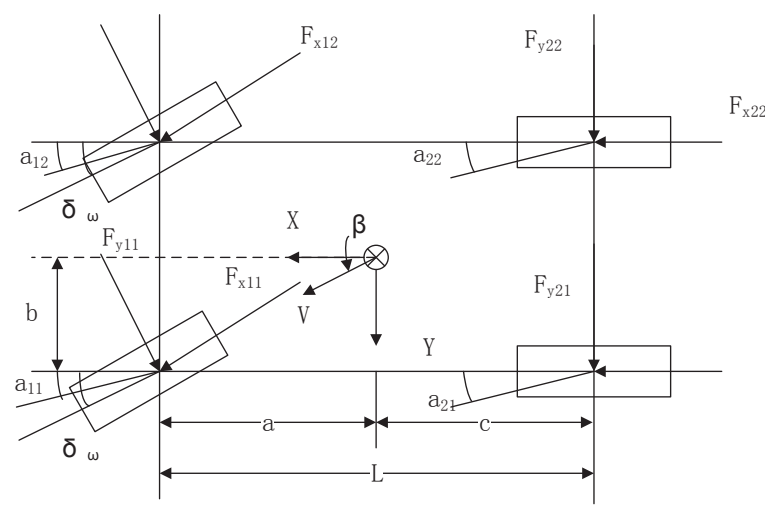

Figure.2 Seven Degree of freedom vehicle model

The significance represented by the parameters described in this paper:

$a$ represents the distance from the center of mass to the front axle;

$b$ represents the distance from the center of mass to the rear axle;

$L$ represents wheelbase;

$c$ represents half track;

$I_{z}$ represents the inertia of the vehicle around the center of mass;

$\theta$ represents the yaw angle of the vehicle;

$\omega_{y}$ represents the yaw rate of the vehicle;

8 , represents front wheel steering angle;

$u$ represents a component of the speed of the vehicle in the direction of the vehicle;

$v$ represents the speed of the vehicle in the direction perpendicular to the direction of the vehicle.

The four wheel motion equation of seven degrees of freedom is as follows:

$$
\begin{aligned}
& m\left(\dot{V}_{x}-V_{y} \dot{\varphi}\right)=\left(F_{x 11}+F_{x 12}\right) \cos \delta-\left(F_{y 11}+F_{y 12}\right) \sin \delta \\
& +F_{x 21}+F_{x 22} \\
& m\left(\dot{V}_{x} \dot{\varphi}+V_{y}\right)=\left(F_{y 11}+F_{y 12}\right) \cos \delta+\left(F_{x 11}+F_{x 12}\right) \sin \delta \\
& +F_{y 21}+F_{y 22}
\end{aligned}
$$




$$
\begin{aligned}
& I_{z} \ddot{\varphi}=-\left[\left(F_{y 11}+F_{y 12}\right) a \cos \delta-\left(F_{y 11}-F_{y 12}\right) c \sin \delta\right] \\
& +\left(F_{y 21}+F_{y 22}\right) b-\left(F_{x 11}+F_{x 12}\right) a \sin \delta \\
& +\left(F_{x 11}-F_{x 12}\right) c \cos \delta+\left(F_{x 21}-F_{x 22}\right) c
\end{aligned}
$$

\subsection{The establishment of four wheel equation}

When the wheel is turning, assume that the following conditions are satisfied: (1) The cornering stiffness of the tire is fixed, and the lateral force is linearly related to the side slip angle; (2) Vehicle runs at constant speed; (3) The steering angle is characterized by low frequency and small angle.

A mathematical model of yaw rate is established according to the seven degree of freedom vehicle motion model:

$$
\begin{gathered}
-\left[\left(F_{y 11}+F_{y 12}\right) a \cos \delta-\left(F_{y 11}-F_{y 12}\right) c \sin \delta\right] \\
+\left(F_{y 21}+F_{y 22}\right) b-\left(F_{x 11}+F_{x 12}\right) a \sin \delta \\
+\left(F_{x 11}-F_{x 12}\right) a \cos \delta+\left(F_{x 21}-F_{x 22}\right) c=M_{z} \\
M_{z} / I_{z}=\ddot{\theta} \\
\int \ddot{\theta} d t=\omega_{r}
\end{gathered}
$$

According to the seven degree of freedom vehicle model, the mathematical model of the sideslip angle of the center of mass is established[5]:

$$
\begin{gathered}
\frac{F_{y}}{m}=a_{y}=\frac{d v}{d t}-u \frac{d \theta}{d t}=\dot{v}-u \omega_{r} \\
\frac{\dot{v}}{u}=\omega_{r}-\frac{a_{y}}{u} \\
\beta=\frac{v}{u}
\end{gathered}
$$

According to the model, the angle of sailing is calculated $\xi$ :

$$
\xi=\frac{v+a \omega_{r}}{u-c \omega_{r}}
$$

The deflection angle of the front axle $\beta_{f}$ and the deflection angle of the rear axle $\beta_{r}$ can be obtained by the longitudinal and vertical velocity components of the front and rear shafts [6]:

$$
\begin{gathered}
\beta_{f}=\frac{v+a \omega_{r}}{u-c \omega_{r}}-\delta \\
\beta_{r}=\frac{v-b \omega_{r}}{u-c \omega_{r}}
\end{gathered}
$$

The deflection angle of the four side tires of the vehicle can be calculated by the equation of motion of the four wheel vehicle:

$$
\begin{aligned}
& \beta_{1}=\frac{v+a \omega_{r}}{u-c \omega_{r}}-\delta \\
& \beta_{2}=\frac{v+a \omega_{r}}{u+c \omega_{r}}-\delta \\
& \beta_{3}=\frac{v-b \omega_{r}}{u-c \omega_{r}} \\
& \beta_{4}=\frac{v-b \omega_{r}}{u+c \omega_{r}}
\end{aligned}
$$

According to the Ackerman steering relation, the turning radius of vehicle center of mass is obtained [7]:

$$
R=\frac{L}{\delta-\beta_{f}+\beta_{r}}
$$

Each wheel's steering radius $\mathrm{R} 1 \sim \mathrm{R} 4$ has strict geometric relationship with centroid steering radius R. And each wheel's velocity can be calculated [8].

\section{Theoretical model simulation analysis}

The seven degree of freedom model is built in Simulink to simulate. In the simulation, we assume that the speed of the car is constant. It is $5.4 \mathrm{~m} / \mathrm{s}$. In the simulation, the sinusoidal input is 15 degrees when the steering wheel angle is amplitude. The wheel speed of the four wheels is also around the speed, showing a sine change. The simulation results are shown in Figure 3.

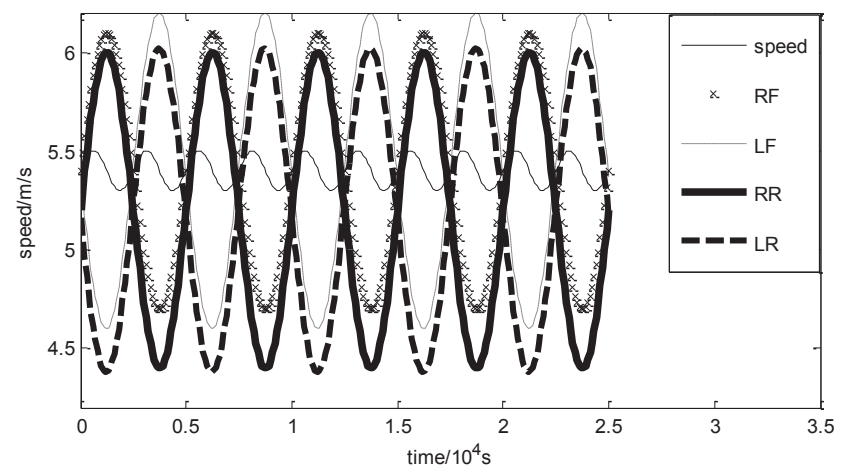

Figure 3. Wheel speed simulation curve.

As can be seen from the diagram, when the vehicle turns left, the left front wheel and left rear wheel speed are less than $5.4 \mathrm{~m} / \mathrm{s}$, and the right front wheel and right rear wheel speed are greater than $5.4 \mathrm{~m} / \mathrm{s}$. When the vehicle turns to the right, the result is the reverse. It can be seen that the algorithm can also calculate the wheel speed under the condition of continuous steering.

\section{Revise under sinusoidal driving condition}

Due to differences in tire wear and road problems, the wheel speeds measured by the four wheels are not equal. 
Therefore, the four wheels should be modified to get the same speed at normal tire pressure.

In the early stage of the task group, the pulse correction under cornering condition has been studied experimentally [9]. Supposing the inside two wheels have equal turning radius, and the radius of the two wheels on the outside is approximately equal. Set the inside wheel radius as $r 1$ and $r 2$. The mean of the two is $r 3$.

Set

$$
k_{i}=\frac{r 3}{R_{i}} \quad i=1,2,3
$$

$k_{i}$ is the correction factor under turn conditions in the formula ${ }^{[4]}$.

$$
v_{w i}=k_{i} * v_{i} \quad i=1,2,3
$$

$v_{w i}$ is the corrected wheel speed under turn conditions. It can call standard turn wheel speed.

After correction, the four wheel speeds eliminate the effects of road wear, wheel wear, and wheel distance between the wheels. Using the three mean comparison method to determine the tire air shortage.

\section{Obtain standard wheel speed under sinusoidal driving condition}

The wheel speed is judged by the three mean comparison method. The three mean comparison method compares the standard wheel speed of a wheel turning standard wheel speed with the average wheel speed of the other three wheels[10].

$$
\begin{gathered}
\bar{v}_{w i}=\frac{1}{3} \sum_{j=1}^{4} v_{j} \quad i=1,2,3 \quad j \neq i \\
\Delta v_{w i}^{\prime}=\left|\frac{v_{i}-v_{w i}^{-}}{-}\right| \quad i=1,2,3,4
\end{gathered}
$$

$\bar{v}_{w i}$ is the standard wheel speed of the other three wheels except the $i$ st wheel, $\Delta v_{w i}^{\prime}$ is the relative error between the standard wheel speed of the $i$ st wheels and the average wheel speed average of the other three wheels. Set $\varepsilon$, is the threshold of the three mean comparison method. And when the relative error $\Delta v_{w i}^{\prime}$ exceeds the threshold, it can determine the lack of gas in the wheel[8].

\section{Road test}

In order to verify the correctness of the theory that the tire pressure anomaly is judged by the seven degree of freedom vehicle model, we have done the pile driving test. Test vehicle selects Havar H6. The tyre model is 225 / 65R17. By the three mean comparison method, the relative errors of the inside and outside sides are different when turning. Due to the wheel speed varies sine, there are two thresholds. Set the threshold value of the inner wheel while turning as $0.06 \%$. And the threshold of the outer wheel is $0.09 \%$. The reference speed is $30 \mathrm{~km} / \mathrm{h}$. The test results are filtered as shown in Figure 4.

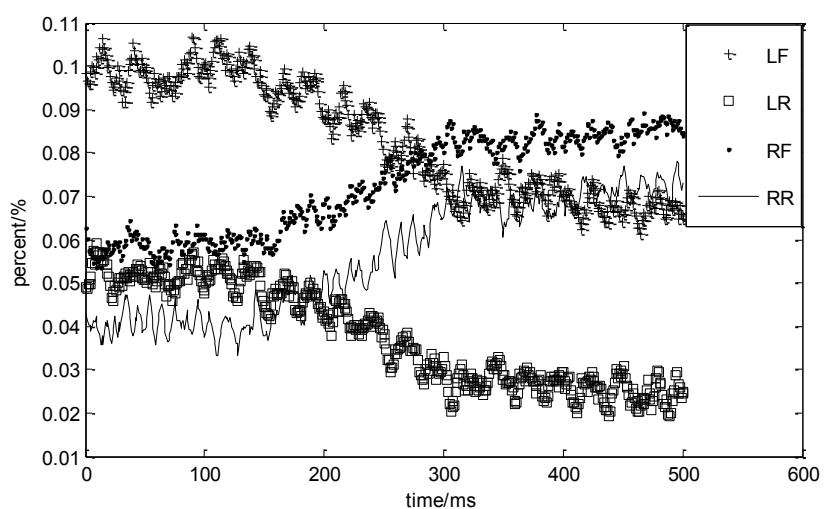

Figure 4. Relative error of wheel speed.

We can see that the left front wheel turning speed relative error exceeds the threshold from the figure, we can judge the left front gas shortage.

\section{Conclusions}

It can be concluded from the experiment that the wheel speed of each wheel varies greatly during cornering, so the wheel speed is judged to be abnormal when cornering, which results in the failure of the tire pressure monitoring system.

The four wheel model of the vehicle takes full advantage of the vehicle inertial unit and steering wheel angle sensor to monitor tire pressure, and when the vehicle is under steering conditions, it can successfully estimate the theoretical speed of each wheel. The seven degree of freedom model takes into account the influence of more parameters, which makes the wheel speed more accurate.

In this paper, wheel speed correction is made by formula (23) and (24), and then the three mean method is used to compare whether the tire pressure is normal or not.

\section{Acknowledge}

This work was support by The national natural science foundation of China (Grant No. 51175450) and Young teachers independent research project topics of Yanshan University, Institute of technology, Class A (Grant No. 14LGA019)

\section{References}

1. National Highway Traffic Safety Administration of USA. Tire pressure maintenance: A statistical investigation [R]. Tire technology International, 2009.

2. Li Liang. Research on state observation and controlling method of vehicle dynamics stability control system [D].Qin Huangdao: Huangdao: Yanshan University, 2008:20-23. 
3. Ren Guo-xin. Study in HIL-simulation technology for ABS/TCS integrative control system of Commercial Vehecle[D]. Jilin University.2008

4. $\mathrm{Hu}$ Jian-jun, Zhao Yu-sheng, Qin Da-tong. Hardware-In-Loop Simulation of HEV System Based on CAN.[J]. China Mechanical Engineering.,2008,19(3): 300-304.

5. Yu Zhuo-ping, Gao Xiao-jie. An overview of the state estimation of the vehicle running $[\mathrm{J}]$. Chinese Journal of Mechanical Engineering, 2009; 45(5), 20-33.

6. Toshihiro Hiraoka, Osamu Nishihara, Hiromitsu Kumamoto. Automatic path-tracking controller of a four-wheel steering vehicle [J]. Vehicle System Dynamics, 2009, 47(10): 1205-1227.

7. Yu Zhi-sheng. The theory of the automobile [M]. Beijing: Machinery Industry Press, 2009:144-146.
8. Zhang Zhe. Monitoring Method of Indirect TPMS under Steering Situation[J]. International Conference on Mechanical, Industrial and Manufacturing (MIME 2016).2016.1,187-190

9. Chen Hui, Gao Bo-Lin, Xu Fan. The summary of the estimation of The vehicle sideslip angle[J]. Chinese Journal of Mechanical Engineering., 2013;49(24):76-94.

10. Han Zong-qi, Liu Quan-you, Wang Li-qiang, Zhang Zhong-xiao, Ju Xue-kun. Study on Tire Pressure Monitoring and Alarming System of Automobile Based on the Comparison among Standard Pulse Numbers[J]. Qin Huangdao: Yanshan University,2010, 21(2) 